\title{
Fan-Planar Graphs: Combinatorial Properties and Complexity Results ${ }^{\star}$
}

\author{
Carla Binucci $^{1}$, Emilio Di Giacomo ${ }^{1}$, Walter Didimo ${ }^{1}$, Fabrizio Montecchiani $^{1}$, \\ Maurizio Patrignani ${ }^{2}$, and Ioannis G. Tollis ${ }^{3}$ \\ 1 Università degli Studi di Perugia, Italy \\ \{carla.binucci, emilio.digiacomo, \\ walter.didimo, fabrizio.montecchiani\}@unipg.it \\ ${ }^{2}$ Università Roma Tre, Italy \\ patrigna@dia.uniroma3.it \\ ${ }^{3}$ Univ. of Crete and Institute of Computer Science-FORTH, Greece \\ tollis@ics.forth.gr
}

\begin{abstract}
In a fan-planar drawing of a graph an edge can cross only edges with a common end-vertex. Fan-planar drawings have been recently introduced by Kaufmann and Ueckerdt, who proved that every $n$-vertex fan-planar drawing has at most $5 n-10$ edges, and that this bound is tight for $n \geq 20$. We extend their result from both the combinatorial and the algorithmic point of view. We prove tight bounds on the density of constrained versions of fan-planar drawings and study the relationship between fan-planarity and $k$-planarity. Also, we prove that testing fan-planarity in the variable embedding setting is NP-complete.
\end{abstract}

\section{Introduction}

There is a growing interest in the study of non-planar drawings of graphs with forbidden crossing configurations. The idea is to relax the planarity constraint by allowing edge crossings that do not affect too much the drawing readability. Among the most popular types of non-planar drawings studied so far we recall: $k$-planar drawings, where an edge can have at most $k$ crossings (see, e.g., [5|8|9|10|16|18|22|26|27|29|30|32]); $k$-quasi-planar drawings, which do not contain $k$ mutually crossing edges (see, e.g., [13:4 15 24|33]); RAC drawings, where edges can cross only at right angles (see, e.g., [19] and [20] for a survey); $A C E_{\alpha}$ drawings [2] and $A C L_{\alpha}$ drawings [6 1421], which are generalizations of RAC drawings; namely, in an $\mathrm{ACE}_{\alpha}$ drawing edges can cross only at an angle that is exactly $\alpha(\alpha \in(0, \pi / 2])$; in an $\mathrm{ACL}_{\alpha}$ drawing edges can cross only at angles that are at least $\alpha$ (see also [20]); fan-crossing free drawings, where there cannot be an edge that crosses two other edges with a common end-vertex [11].

Given a desired type $T$ of non-planar drawing with forbidden crossing configurations, a classical combinatorial problem is to establish bounds on the maximum

\footnotetext{
^ Research supported in part by the MIUR project AMANDA "Algorithmics for MAssive and Networked DAta", prot. 2012C4E3KT_001. This work started at the Bertinoro Workshop on Graph Drawing 2014. We thank Michael Kaufmann and Torsten Ueckerdt for suggesting the study of fan-planar graphs during the workshop. We also thank all the participants of the workshop for the useful discussions on this topic.
} 


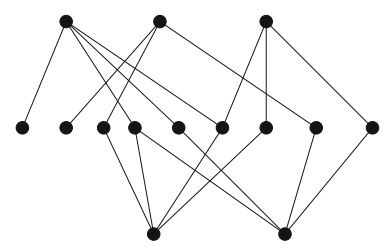

(a)

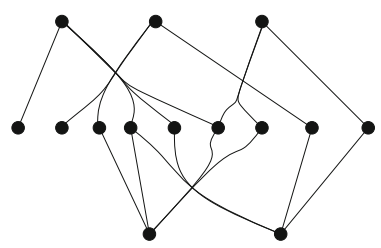

(b)

Fig. 1. (a) A fan-planar drawing of a graph $G$ with 12 crossings; (b) A confluent drawing of $G$ with 3 crossings

number of edges that a drawing of type $T$ can have; this problem is usually dubbed a Turán-type problem, and several tight bounds have been proved for the types of drawings mentioned above, both for straight-line and for polyline edges (see,

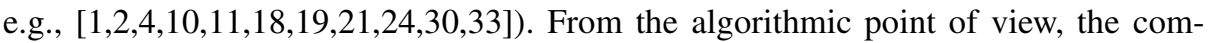
plexity of testing whether a graph $G$ admits a drawing of type $T$ is one of the most interesting. Also for this problem several results have been shown, both in the variable and in the fixed embedding setting (see, e.g., [8|12|13|25|26 29] ).

In this paper we investigate fan-planar drawings of graphs, in which an edge cannot cross two independent edges, i.e., an edge can cross several edges provided that they have a common end-vertex. Fan-planar drawings have been recently introduced by Kaufmann and Ueckerdt [28]; they proved that every $n$-vertex graph without loops and multiple edges that admits a fan-planar drawing has at most $5 n-10$ edges, and that this bound is tight for $n \geq 20$. Fan-planar drawings are on the opposite side of fan-crossing free drawings mentioned above. Besides its intrinsic theoretical interest, we observe that fan-planarity can be also used for creating drawings with few edge crossings in a confluent drawing style (see, e.g., [17/23]). For example, Fig. 1(a)] shows a fan-planar drawing $\Gamma$ with 12 crossings; Fig. 1(b) shows a new drawing with just 3 crossings obtained from $\Gamma$ by bundling crossing "fans".

We prove both combinatorial properties and complexity results related to fan-planar drawings of graphs. The main contributions of our work are as follows:

(i) We study the density of constrained versions of fan-planar drawings (Sec. 3), namely outer fan-planar drawings, where all vertices must lie on the external boundary of the drawing, and 2-layer fan-planar drawings, where vertices are placed on two distinct horizontal lines and edges are vertically monotone lines. We prove tight bounds for the edge density of these drawings. Namely, we show that $n$-vertex outer fan-planar drawings have at most $3 n-5$ edges (a tight bound for $n \geq 5$ ), and that $n$-vertex 2 layer fan-planar drawings have at most $2 n-4$ edges (a tight bound for $n \geq 3$ ). We remark that outer and 2-layer non-planar drawings have been previously studied in the 1-planarity setting [8 18 26] and in the RAC planarity setting [12]13].

(ii) Since general fan-planar drawable graphs have at most $5 n-10$ edges and the same bound holds for 2-planar drawable graphs [30], we investigate the relationship between these two graph classes. More in general, we are able to prove that in fact for any $k \geq 2$ there exist fan-planar drawable graphs that are not $k$-planar, and vice versa (Sec.4). 
(iii) Finally, we show that testing whether a graph admits a fan-planar drawing in the variable embedding setting is NP-complete (Sec. 5).

Preliminaries are in Sec. 2. Open problems can be found in Sec.6 For space reasons some proofs are sketched or omitted.

\section{Preliminary Definitions and Results}

A drawing $\Gamma$ of a graph $G$ maps each vertex to a distinct point of the plane and each edge to a simple Jordan arc between the points corresponding to the end-vertices of the edge. For a subgraph $G^{\prime}$ of $G$, we denote by $\Gamma\left[G^{\prime}\right]$ the restriction of $\Gamma$ to $G^{\prime}$. Throughout the paper we consider only simple graphs, i.e., graphs with neither multiple edges nor self-loops; also we only consider simple drawings, i.e., drawings such that the arcs representing two edges have at most one point in common, which is either a common end-vertex or a common interior point where the two arcs properly cross each other.

For each vertex $v$ of $G$, the set of edges incident to $v$ is called the fan of $v$. Clearly, each edge $(u, v)$ of $G$ belongs to the fan of $u$ and to the fan of $v$ at the same time. Two edges that do not share a vertex are called independent edges; two independent edges always belong to distinct fans. A fan-planar drawing $\Gamma$ of $G$, is a drawing of $G$ such that: $(a)$ no edge is crossed by two independent edges; $(b)$ there are not two adjacent edges $(u, v),(u, w)$ that cross an edge $e$ from different "sides" while moving from $u$ to $v$ and from $u$ to $w$. The forbidden configurations $(a)$ and $(b)$ are depicted in Fig. 2(a) and Fig. 2(b), respectively. Figures 2(c) and 2(d) show two allowed configurations of a fan-planar drawing. A fan-planar graph is a graph that admits a fan-planar drawing.

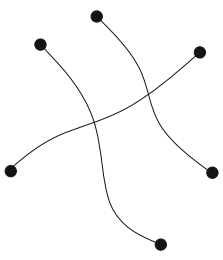

(a)

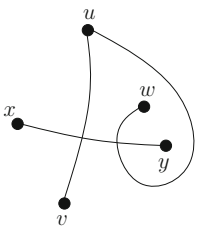

(b)

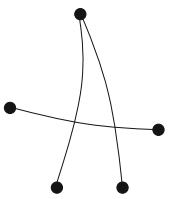

(c)

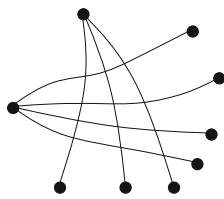

(d)

Fig. 2. (a)-(b) Forbidden configurations in a fan-planar drawing: (c)-(d) Allowed configurations in a fan-planar drawing

The next property immediately follows from the definition of fan-planar drawings.

Property 1. A fan-planar drawing does not contain 3-mutually crossing edges.

Let $\Gamma$ be a non-planar drawing of $G$; the planar enhancement $\Gamma^{\prime}$ of $\Gamma$ is the drawing obtained from $\Gamma$ by replacing each crossing point with a dummy vertex. The boundary of each face $f^{\prime}$ of $\Gamma^{\prime}$ consists of a sequence of real and dummy vertices; the connected region $f$ of the plane that corresponds to $f^{\prime}$ in $\Gamma$ consists of a sequence of vertices and crossing points. For simplicity we call $f$ a face of $\Gamma$. The outer face of $\Gamma$ is the face corresponding to the outer face of $\Gamma^{\prime}$. A fan-planar drawing of $G$ with all vertices on the 
outer face is called an outer fan-planar drawing of $G$. Observe that the configuration in Fig. 2(b) cannot occur in a drawing with all vertices on the outer face; hence, a drawing is outer fan-planar if and only if all vertices are on the outer face and it does not contain an edge crossed by two independent edges. An outer fan-planar graph is a graph that admits an outer fan-planar drawing. An outer fan-planar graph $G$ is maximal, if no edge can be added to $G$ without loosing the property that $G$ remains outer fan-planar. An outer fan-planar graph $G$ with $n$ vertices is maximally dense if it has the maximum number of edges among all outer fan-planar graphs with $n$ vertices. If $G$ is maximally dense then it is also maximal, but not vice versa. The following property holds.

Lemma 1. Let $G=(V, E)$ be a maximal outer fan-planar graph and let $\Gamma$ be an outer fan-planar drawing of $G$. The outer face of $\Gamma$ does not contain crossing points, i.e., it consists of $|V|$ uncrossed edges.

Given an outer fan-planar drawing $\Gamma$ of a maximal outer fan-planar graph $G$, the edges of $G$ on the external boundary of $\Gamma$ will be also called the outer edges of $\Gamma$.

A 2-layer fan-planar drawing is a fan-planar drawing such that: $(i)$ each vertex is drawn on one of two distinct horizontal lines, called layers; $(i i)$ each edge connects vertices of different layers and it is drawn as a vertical monotone curve. By definition, a 2-layer fan-planar drawing is also an outer fan-planar drawing. A 2-layer fan-planar graph is a graph that admits a 2-layer fan planar drawing.

\section{Density of Outer and 2-layer Fan-Planar Graphs}

We first prove that an $n$-vertex outer fan-planar graph $G$ has at most $3 n-5$ edges. Then we describe how to construct outer fan-planar graphs with $n$ vertices and $3 n-5$ edges.

Let $G$ be a graph and let $\Gamma$ be a drawing of $G$. The crossing graph of $\Gamma$, denoted as $\operatorname{CR}(\Gamma)$, is a graph having a vertex for each edge of $G$ and an edge between any two vertices whose corresponding edges cross in $\Gamma$. A cycle of $\operatorname{CR}(\Gamma)$ of odd length will be called an odd cycle of $\mathrm{CR}(\Gamma)$; similarly, an even cycle of $\operatorname{CR}(\Gamma)$ is a cycle of even length. In order to prove the $3 n-5$ upper bound, we can assume that $G$ is a maximally dense outer fan-planar graph. We start by proving some interesting combinatorial properties of $G$ related to the cycles of the crossing graph of $G$.

Lemma 2. Let $G=(V, E)$ be a maximal outer fan-planar graph with $n=|V|$ vertices and $m=|E|$ edges. Let $\Gamma$ be an outer fan-planar drawing of $G$. If $\operatorname{CR}(\Gamma)$ does not have odd cycles then $m \leq 3 n-6$.

Proof. If $\mathrm{CR}(\Gamma)$ does not contain odd cycles, then it is bipartite and its vertices can be partitioned into two independent sets $W_{1}$ and $W_{2}$. Since by Lemma 1 the outer edges of $\Gamma$ are not crossed, they correspond to $n$ isolated vertices in $\operatorname{CR}(\Gamma)$. We can arbitrarily assign all these vertices to the same set, say $W_{1}$. Denote by $E_{i}$ the set of edges of $G$ corresponding to the vertices of $W_{i}(i \in\{1,2\})$. Clearly, $E_{1}$ and $E_{2}$ partition the set $E$. Since no two edges of $E_{i}$ cross in $\Gamma$, then the two subgraphs $G_{1}=\left(V, E_{1}\right)$ and $G_{2}=\left(V, E_{2}\right)$ are outerplanar graphs, where $\left|E_{1}\right| \leq 2 n-3$ and $\left|E_{2}\right| \leq 2 n-3-n$. Thus, $m=|E|=\left|E_{1}\right|+\left|E_{2}\right| \leq 3 n-6$.

The next lemma shows that the length of any odd cycle of $\mathrm{CR}(\Gamma)$ is at most 5 . 
Lemma 3. Let $G$ be a maximally dense outer fan-planar graph with $n$ vertices and let $\Gamma$ be an outer fan-planar drawing of $G . \mathrm{CR}(\Gamma)$ does not contain odd cycles of length greater than 5 .

Proof. Let $C$ be an odd cycle of length $\ell$ in $\mathrm{CR}(\Gamma)$. Let $E(C)=\left\{e_{0}=\right.$ $\left.\left(u_{0}, v_{0}\right), \ldots, e_{\ell-1}=\left(u_{\ell-1}, v_{\ell-1}\right)\right\}$ be the set of $\ell$ edges of $G$ corresponding to the vertices of $C$, such that $e_{i}$ crosses $e_{i+1}$ for $i=0, \ldots, l-1$, where indices are taken modulo $\ell$. Recall that all vertices of $G$ are on the outer face of $\Gamma$, which implies that the end-vertices of the edges in $E(C)$ are encountered in the following order when walking clockwise on the boundary of the outer face of $\Gamma: u_{i}$ precedes $v_{i-1}$ and $v_{i}$ precedes $u_{i+2}$ (see, e.g., Fig. 3(a)). Furthermore, vertices $v_{i}$ and $u_{i+2}$ must coincide, for $i=0, \ldots, \ell-1$. Indeed, if $v_{i}$ and $u_{i+2}$ are distinct, for some $i=0, \ldots, \ell-1$, then edge $e_{i+1}$ is crossed by two independent edges (i.e., $e_{i}$ and $e_{i+2}$ ), which contradicts the hypothesis that $\Gamma$ is fan-planar. See also Fig. 3(a) Thus, we have that $u_{i}$ precedes $u_{i+1}$ while walking clockwise on the boundary of the outer face of $\Gamma$, for $i=0, \ldots, \ell-1$, as shown in Fig. 3(b). Moreover, it can be seen that the edges in $E(C)$ are not crossed by any edge not in $E(C)$, as otherwise the drawing would not be fan-planar.

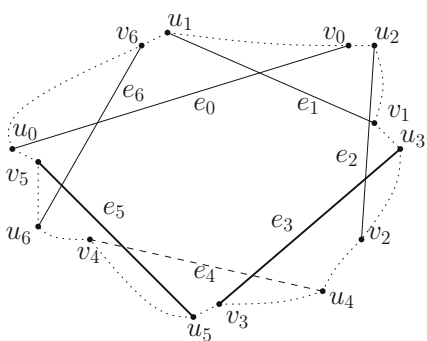

(a)

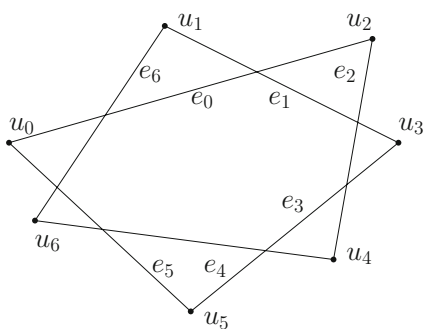

(b)

Fig. 3. Illustration for the proof of Lemma3 (a) An edge set $E(C)$ with $\ell=7$. If $v_{3}$ and $u_{5}$ do not coincide, $e_{4}$ (dashed) is crossed by the two independent edges $e_{3}$ and $e_{5}$ (in bold). (b) $E(C)$ with $\ell=7$, where $v_{i}$ coincides with $u_{i+2}$, for $i=0, \ldots, 7$.

Now, suppose by contradiction that $\ell$ is odd and greater than 5 (see Fig. 3(b)). Consider a vertex $u_{i}$, for some $i=0, \ldots, \ell-1$, and denote by $\bar{V}$ the set of vertices encountered between $u_{i+3}$ and $u_{i-3}$ while walking clockwise on the boundary of the outer face of $\Gamma$ (including $u_{i+3}$ and $u_{i-3}$ ). Vertex $u_{i}$ cannot be adjacent to any vertex in $\bar{V}$. Namely, if an edge $e=\left(u_{i}, u_{j}\right)$ existed, for some $u_{j} \in \bar{V}$, then it would be crossed by the two independent edges $e_{i-1}$ and $e_{j-1}$. Thus, removing $e_{i-1}$ from $\Gamma$, one can suitably connect $u_{i}$ to all the vertices in $\bar{V}$, still obtaining a fan-planar drawing $\Gamma^{*}$ with $n$ vertices. Since the size of $\bar{V}$ is $\ell-5$, and $\ell \geq 7$ by assumption, then $\Gamma^{*}$ has at least two edges more than $\Gamma$, which contradicts the hypothesis that $G$ is maximally dense.

The following corollary is a consequence of Lemma 3 and Property 1.

Corollary 1. Let $G$ be a maximally dense outer fan-planar graph. Any odd cycle in the crossing graph of a fan-planar drawing of $G$ has exactly length 5. 
The next lemma claims that odd cycles in the crossing graph correspond to $K_{5}$ (the proof uses similar arguments as the proof of Lemma3.

Lemma 4. Let $G$ be a maximally dense outer fan-planar graph and let $\Gamma$ be an outer fan-planar drawing of $G$. If $\mathrm{CR}(\Gamma)$ contains a cycle $C$ of length 5 , then the subgraph of $G$ induced by the end-vertices of the edges corresponding to the vertices of $C$ is $K_{5}$.

We now prove the upper bound on the density of outer fan-planar graphs.

Lemma 5. Let $G$ be a maximally dense outer fan-planar graph with $n$ vertices and $m$ edges. Then $m \leq 3 n-5$ edges.

Proof. Let $\Gamma$ be an outer fan-planar drawing of $G$. We first claim that $G$ is biconnected. Suppose by contradiction that $G$ is not biconnected, and let $C_{1}$ and $C_{2}$ be two distinct biconnected components of $G$ that share a cut-vertex $v$. Let $u$ be the first vertex of $G$ encountered while moving from $v$ clockwise on the external boundary of $\Gamma\left[C_{1}\right]$, and let $w$ be the first vertex encountered while moving from $v$ counterclockwise on the external boundary of $\Gamma\left[C_{2}\right]$. One can suitably add edge $(u, w)$ in $\Gamma$, still getting an outer fan-planar drawing, which contradicts the hypothesis that $G$ is maximally dense.

Now, by Corollary $1 \mathrm{CR}(\Gamma)$ can only have either even cycles or cycles of length 5 . Also, by Lemma 4 , every cycle of length 5 in $\mathrm{CR}(\Gamma)$ corresponds to a subset of edges whose end-vertices induce $K_{5}$. We prove the statement by induction on the number $h$ of $K_{5}$ subgraphs in $G$.

Base Case. If $h=0$ then, by Lemma 2, $G$ has at most $3 n-6$ edges.

Inductive Case. Suppose by induction that the claim is true for $h \geq 0$, and suppose $G$ contains $h+1$ subgraphs that are $K_{5}$. Let $G^{*}$ be one of these $h+1$ subgraphs. Let $e=(u, v)$ be an edge on the outer face of $\Gamma\left[G^{*}\right]$ that is not on the outer face of $\Gamma$. Vertices $u$ and $v$ are a separation pair of $G$, otherwise there would be a vertex of $G$ that is not on the outer face of $\Gamma$, which is impossible because $\Gamma$ is an outer fan-planar drawing by hypothesis. Hence, we can split $G$ into two biconnected subgraphs that share only edge $e$, one of them containing $G^{*}$. Let $G_{1}, G_{2}, \ldots, G_{k}(k \leq 5)$ be the biconnected subgraphs of $G$ distinct from $G^{*}$ such that each $G_{i}$ shares exactly one edge with $G^{*}$. Each $G_{i}(i=1,2, \ldots, k)$ contains at most $h$ subgraphs that are $K_{5}$, and therefore it has at most $3 n_{i}-5$ edges by induction, where $n_{i}$ denotes the number of vertices of $G_{i}$. On the other hand, $G^{*}$ has $3 n^{*}-5=10$ edges, where $n^{*}=5$ is the number of vertices of $G^{*}$. It follows that $m \leq 3\left(n^{*}+n_{1}+\cdots+n_{k}\right)-5(k+1)-k(k \leq 5)$. Since $n^{*}+n_{1}+\cdots+n_{k} \leq n+2 k$ we have $m \leq 3(n+2 k)-5(k+1)-k=3 n-5$.

The existence of an infinite family of outer fan-planar graphs that match the $3 n-5$ bound is proved in the next lemma. Refer to Fig. 4 for an illustration.

Lemma 6. For any integer $h \geq 1$ there exists an outer fan-planar graph $G$ with $n=$ $3 h+2$ vertices and $m=3 n-5$ edges.

Proof. Consider $h$ graphs $X_{1}, \ldots, X_{h}$, such that each $X_{i}$ is a $K_{5}$ graph, for $i=$ $1, \ldots, h$. We now describe how to construct $G$. The idea is to "glue" $X_{1}, \ldots, X_{h}$ together in such a way that they share single edges one to another. The proof is by induction on the number of merged graphs. Denote by $G_{i}$ the graph obtained after merging 


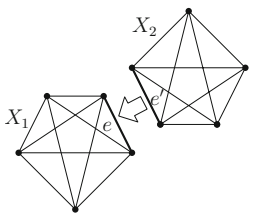

(a)

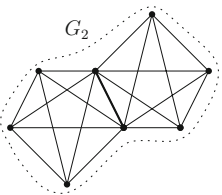

(b)

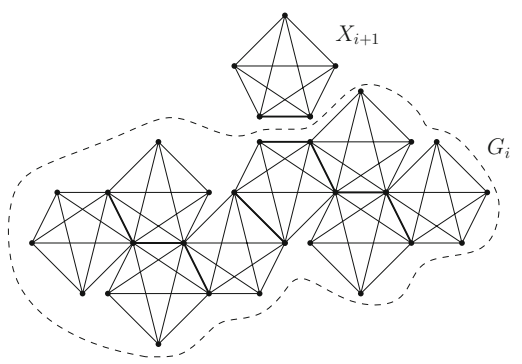

(c)

Fig. 4. Illustration for the proof of Lemma 6 (a) $X_{1}$ and $X_{2}$ before being merged. (b) Merging $X_{1}$ and $X_{2}$ into $G_{2}$. (c) $G_{i}$ and $X_{i+1}$, the bold edges are used for merging.

$X_{1}, \ldots, X_{i}$, for $1<i \leq h$. We prove by induction that $G_{i}$ respects the following invariants: (I1) it is an outer fan-planar graph; (I2) it has $n_{i}=3 i+2$ vertices and $m_{i}=3 n_{i}-5$ edges. In the base case $i=2$, we merge $G_{1}=X_{1}$ to $X_{2}$ as follows. Pick an edge $e$ on the outer face of $X_{1}$ and an edge $e^{\prime}$ on the outer face of $X_{2}$. Merge $X_{1}$ and $X_{2}$ by identifying $e$ with $e^{\prime}$, see also Figs. 4(a) and 4(b) The new graph $G_{2}$ is clearly an outer fan-planar graph with $n_{2}=5+5-2=8$ vertices and $m_{2}=10+10-1=19$ edges. Thus, the two invariants hold. In the inductive case, suppose we constructed $G_{i}$ for $2<i<h$ and we want to attach $X_{i+1}$ (see also Fig. 4(c)). Pick any edge $e$ on the outer face of $G_{i}$ and any edge $e^{\prime}$ on the outer face of $X_{i+1}$. Merge the two graphs in the same way as done in the base case. It is immediate to see that (I1) holds. Also, $n_{i+1}=n_{i}+3$ and $m_{i+1}=m_{i}+9$. Since by induction $m_{i}=3 n_{i}-5$, then $m_{i+1}=3 n_{i}-5+9=3 n_{i+1}-5$.

Lemmas 5 and 6 imply the following theorem.

Theorem 1. An outer fan-planar graph with $n$ vertices has at most $3 n-5$ edges, and this bound is tight for $n \geq 5$.

An obvious consequence of Theorem 1 and of the definition of outer fan-planar graphs that are maximally dense is the following fact.

Corollary 2. Every maximally dense outer fan-planar graph with $n=3 h+2$ vertices $(h \geq 1)$ has $3 n-5$ edges.

Concerning 2-layer fan planar graphs, we already observed that a 2-layer fan planar graph $G$ is an outer fan-planar graph. Also, since all vertices on the same layer form an independent set, $G$ is bipartite.

Theorem 2. A 2-layer fan-planar graph with $n$ vertices has at most $2 n-4$ edges, and this bound is tight for $n \geq 3$.

Proof. Let $G$ be a maximally dense 2-layer fan-planar graph with $n$ vertices and $m$ edges, and let $\Gamma$ be a 2-layer fan-planar drawing of $G$. Let $V_{1}=\left\{v_{1}, \ldots, v_{n_{1}}\right\}$ and 
$V_{2}=\left\{v_{n_{1}+1}, \ldots, v_{n}\right\}$ the two independent sets of vertices of $G$. Suppose that in $\Gamma$ $v_{i}$ precedes $v_{i+1}$ along the layer of $V_{1}$ (for $i=1, \ldots, n_{1}-1$ ), and $v_{j}$ precedes $v_{j+1}$ along the layer of $V_{2}$ (for $j=n_{1}+1, \ldots, n-1$ ). Construct from $G$ a super-graph $G^{*}$, by adding an edge $\left(v_{i}, v_{i+1}\right)$, for $i=1, \ldots, n_{1}-1$, and an edge $\left(v_{j}, v_{j+1}\right)$, for $j=n_{1}+1, \ldots, n$. Graph $G^{*}$ is still outer fan-planar. Moreover, since $G$ does not contain a $K_{5}$ subgraph (because it is bipartite), also $G^{*}$ does not contain a $K_{5}$ subgraph, as otherwise at least three vertices of the same layer in $G$ should form a 3-cycle in $G^{*}$ (which does not happen by construction). Thus, by Lemma 3 and Property 1 , the crossing graph of any outer fan-planar drawing of $G^{*}$ contains only even cycles. Hence, denoted as $m^{*}$ the number of edges of $G^{*}$, by Lemma 2 we have $m^{*} \leq 3 n-6$, and therefore $m=m^{*}-(n-2) \leq 2 n-4$. A family of 2-layer fan-planar graphs with $2 n-4$ edges (for $n \geq 3$ ) are the complete bipartite graphs $K_{2, n-2}$.

\section{Fan-Planar and $k$-planar Graphs}

A $k$-planar drawing is a drawing where each edge is crossed at most $k$ times, and a $k$-planar graph is a graph that admits a $k$-planar drawing. Clearly, every 1-planar graph is also a fan-planar graph. Also, both the maximum number of edges of fanplanar graphs [28] and the maximum number of edges of 2-planar graphs [30] have been shown to be $5 n-10$. Thus it is natural to ask what is the relationship between fanplanar and 2-planar graphs. More in general, we prove that there are fan-planar graphs that are not $k$-planar, for any $k \geq 1$, and that there are $k$-planar graphs (for $k>1$ ) that are not fan-planar. The existence of fan-planar graphs that are not $k$-planar is proved with a counting argument on the minimum number of crossings of graph drawings. The crossing number $\operatorname{cr}(G)$ of $G$ is the smallest number of crossings required in any drawing of $G$. We give the following.

Theorem 3. For any integer $k \geq 1$ there is a graph that is fan-planar but not $k$-planar.

Proof. Consider the complete 3-partite graph $K_{1,3, h}$. This graph is fan-planar for every $h \geq 1$ (see Fig. 5(a)]. It is known that $\operatorname{cr}\left(K_{1,3, h}\right)=2\left\lfloor\frac{h}{2}\right\rfloor\left\lfloor\frac{h-1}{2}\right\rfloor+\left\lceil\frac{h}{2}\right\rceil$ [7|31]. For $h=4 k+2$, we have $\operatorname{cr}\left(K_{1,3,4 k+2}\right)=2\left\lfloor\frac{4 k+2}{2}\right\rfloor\left\lfloor\frac{4 k+1}{2}\right\rfloor+\left\lceil\frac{4 k+2}{2}\right\rceil=4 k(2 k+1)+2 k+$ $1=8 k^{2}+6 k+1$. Thus, in every drawing of $K_{1,3,4 k+2}$ there are at least $8 k^{2}+6 k+1$ crossings. On the other hand, in a $k$-planar drawing there can be at most $\frac{\mathrm{km}}{2}$ crossings, where $m$ is the number of edges in the drawing. Since $K_{1,3,4 k+2}$ has $16 k+11$ edges, to be $k$-planar it should admit a drawing with at most $\frac{k m}{2}=\frac{k(16 k+11)}{2}=8 k^{2}+\frac{11}{2} k$ crossings. Since $6 k+1>\frac{11}{2} k$ for every $k \geq 1, K_{1,3,4 k+2}$ is not $k$-planar.

To prove that for any $k>1$ there are $k$-planar graphs that are not fan-planar (Theorem 4), we first give a technical result (Lemma 7), which will be also reused in Sec. 5. Let $\Gamma$ be a fan-planar drawing of a graph. We may regard crossed edges of $\Gamma$ as composed by fragments, where a fragment is the portion of the edge that is between two consecutive crossings or between one of the two end-vertices of the edge and the first crossing encountered while moving along the edge towards the other end-vertex. An edge that is not crossed does not have any fragment. Figure 5(b) shows a fan-planar drawing of the $K_{7}$ graph and Fig. 5(c) shows the fragments of the drawing in Fig. 5(b). 


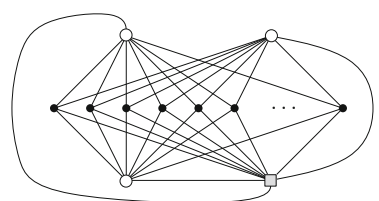

(a)

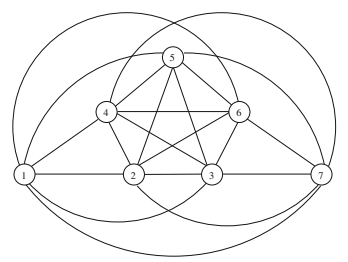

(b)

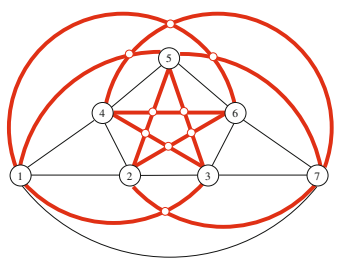

(c)

Fig. 5. (a) A fan-planar drawing of $K_{1,3, h}$. (b) A fan-planar drawing of the $K_{7}$ graph. (c) The fragments of the fan-planar drawing in (b) are the thicker lines.

We consider two fragments adjacent if they share a common crossing or a common end-vertex. The next lemma provides an interesting and useful property.

Lemma 7. In any fan-planar drawing of the $K_{7}$ graph, any pair of vertices is joined by a sequence of adjacent fragments.

Theorem 4. For any integer $k>1$ there is a graph that is $k$-planar but not fan-planar.

Proof sketch: Since 2-planar graphs are also $k$-planar graphs, for $k>1$, we can prove that there is a 2-planar graph that is not fan-planar. Let $G^{\prime}$ be a graph consisting of a cycle $C=\left(v_{1}, v_{2}, \ldots, v_{10}\right)$ and of the edges $\left(v_{1}, v_{4}\right),\left(v_{5}, v_{10}\right),\left(v_{6}, v_{9}\right)$ (see Fig. 6(a)). Let $G^{\prime \prime}$ be the graph obtained from $G^{\prime}$ by replacing each edge $\left(v_{i}, v_{j}\right)(1 \leq i, j \leq 10)$ with a copy of $K_{7}$, whose vertices are denoted as $u_{1}, u_{2}, \ldots, u_{7}$, so that $v_{i}=u_{1}$ and $v_{j}=u_{7}$ (see Fig. 6(b)]. The copy of $K_{7}$ that replaces $\left(v_{i}, v_{j}\right)$ is denoted as $K_{7}^{i, j}$. Let $G$ be the graph obtained from $G^{\prime \prime}$ by adding the edges $\left(v_{1}, v_{7}\right),\left(v_{2}, v_{6}\right),\left(v_{3}, v_{9}\right),\left(v_{4}, v_{8}\right)$ (see Fig. 6(c) p. $G$ is 2-planar (planarly embed $G^{\prime}$ as shown in Fig. 6(a)). Construct a drawing $\Gamma$ of $G$ by replacing each edge of $G^{\prime}$ with a drawing of $K_{7}^{i, j}$ like that in Fig. 5(b) (see Fig. 6(b)], and draw the edges $\left(v_{1}, v_{7}\right),\left(v_{2}, v_{6}\right),\left(v_{3}, v_{9}\right),\left(v_{4}, v_{8}\right)$ inside $C$ as in Fig. 6(c) $\Gamma$ is 2-planar. To prove that $G$ is not fan-planar note that, by Lemma7, each $K_{7}^{i, j}(1 \leq i, j \leq 10)$ has a sequence of fragments leading from $v_{i}=u_{1}$ to $v_{j}=u_{7}$, which we call spine. In any fan-planar drawing of $G$, this spine cannot be crossed by edges that do not belong to $K_{7}^{i, j}$ (otherwise there would be an edge crossed by two independent edges). Thus, every $K_{7}^{i, j}$ is not "traversed" by external edges, and this forces $\left(v_{1}, v_{7}\right),\left(v_{2}, v_{6}\right),\left(v_{3}, v_{9}\right),\left(v_{4}, v_{8}\right)$ to violate fan-planarity, as in Fig.6(c),

\section{Complexity of the Fan-Planarity Testing Problem}

We exploit the results of Secs. 3 and 4 to prove that testing whether a graph is fanplanar in the variable embedding setting is NP-complete. We call this problem the fanplanarity testing. We use a reduction from the 1-planarity testing, which is NP-complete in the variable embedding setting [25[29]. The 1-planarity testing asks whether a given graph admits a 1-planar drawing. We prove the following.

Theorem 5. Fan-planarity testing is NP-complete. 


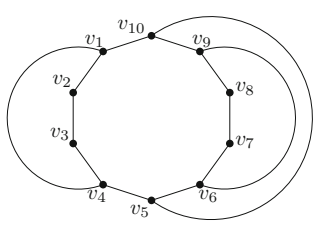

(a)

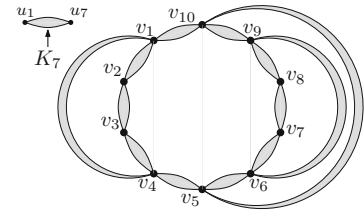

(b)

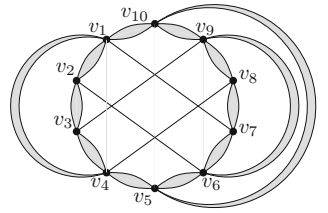

(c)

Fig. 6. (a)-(c) Illustration for the proof of Theorem 4 . (a) the graph $G^{\prime}$; (b) the graph $G^{\prime \prime}$; (c) the graph $G$

Proof. Similarly to [34], a non-deterministic algorithm to test if a graph admits a fanplanar drawing with $k$ crossings considers all possible $k$ pairs of edges that cross (and the order of the crossings along the edges), discards the configurations where an edge crosses more than one fan, replaces crossings with dummy vertices, and tests the obtained graph for planarity. Then the problem belongs to NP.

We now prove the hardness. Given an instance $G=(V, E)$ of the 1-planarity testing we build an instance $G_{f}=\left(V_{f}, E_{f}\right)$ of the fan-planarity testing by replacing each edge $(u, v) \in E$ with two $K_{7}$ graphs with vertices $u=u_{1}, u_{2}, \ldots, u_{7}$ and $v=v_{1}, v_{2}, \ldots, v_{7}$, called attachment gadgets and joined by a spanning edge $\left(u_{7}, v_{7}\right)$ (see Fig. 7 for an illustration). $G_{f}=\left(V_{f}, E_{f}\right)$ can be constructed in polynomial time, having $\left|V_{f}\right|=|V|+|E| \times 12$ vertices and $\left|E_{f}\right|=|E| \times 43$ edges, where $|E| \times 42$ of them belong to the attachment gadgets and the remaining $|E|$ are spanning edges that join different attachment gadgets. We show that $G$ is 1-planar if and only if $G_{f}$ is fan-planar. If $G$ admits a 1-planar drawing, replace each edge $(u, v)$ of $G$ with two fanplanar drawings of $K_{7}$ like those depicted in Fig. 5(b) and with edge $\left(u_{7}, v_{7}\right)$, in such a way that the possible crossing of $(u, v)$ occurs on $\left(u_{7}, v_{7}\right)$. The obtained drawing of $G_{f}$ is fan-planar since each attachment gadget has a fan-planar drawing and each spanning edge has at most one crossing. Conversely, suppose $G_{f}$ admits a fan-planar drawing $\Gamma_{f}$. By Lemma 7 for any attachment gadget of $G_{f}$ attached to vertex $u$, there is at least a sequence of fragments leading from $u=u_{1}$ to $u_{7}$. As in the proof of Theorem 4 , call such a sequence of fragments the spine of the attachment gadget. Delete from $\Gamma_{f}$ all fragments except those in the spines. Delete from $\Gamma_{f}$ all uncrossed edges except the spanning edges. Remove also isolated vertices. A drawing $\Gamma$ of $G$ is obtained, where the drawing of edge $(u, v)$ is given by the spine from $u=u_{1}$ to $u_{7}$, the spanning edge $\left(u_{7}, v_{7}\right)$, and the spine from $v_{7}$ to $v_{1}=v$. Observe that, $u \neq v$, as otherwise there would be a self-loop in $G$. We claim that $\Gamma$ is a 1-planar drawing of $G$. Indeed, fragments in the spines can not be crossed by any other fragment or spanning edge of $\Gamma_{f}$. It follows that spanning edges can cross only among themselves in $\Gamma_{f}$. However, they can cross only once, as they are a matching of $G_{f}$ and $\Gamma_{f}$ is fan-planar. Hence, $\Gamma$ is a 1-planar drawing, but not necessarily simple; indeed, it may happen that two crossing edges $(u, v)$ and $(w, z)$ in $\Gamma$ share an end-vertex, say $u=w$ (this happens when in $\Gamma_{f}$ there are two crossing spanning edges of two $K_{7}$ attached to $u$ ). The crossing between $(u, v)$ and $(u, z)$ in $\Gamma$ can be easily removed by rerouting the edges (see Fig. 7(c)]. 


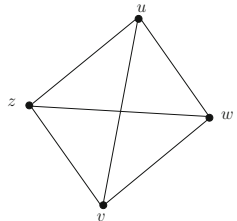

(a)

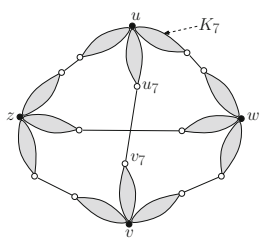

(b)

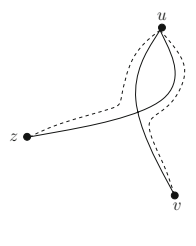

(c)

Fig. 7. Illustration of the reduction used in Theorem 5 (a) An instance $G$ of 1-planarity testing; (b) The reduced instance $G_{f}$ of fan-planarity testing. (c) Two adjacent edges of $G$ that cross one to another in $\Gamma$; the crossing can be removed by rerouting the two edges as shown by the dashed lines.

\section{Open Problems}

We suggest two questions: $(i)$ What is the minimum number of edges of maximal fanplanar graphs? (ii) Can we efficiently recognize maximally dense fan-planar graphs?

\section{References}

1. Ackerman, E.: On the maximum number of edges in topological graphs with no four pairwise crossing edges. Discrete \& Computational Geometry 41(3), 365-375 (2009)

2. Ackerman, E., Fulek, R., Tóth, C.D.: Graphs that admit polyline drawings with few crossing angles. SIAM J. on Discrete Mathematics 26(1), 305-320 (2012)

3. Ackerman, E., Tardos, G.: On the maximum number of edges in quasi-planar graphs. J. of Combinatorial Theory, Series A 114(3), 563-571 (2007)

4. Agarwal, P.K., Aronov, B., Pach, J., Pollack, R., Sharir, M.: Quasi-planar graphs have a linear number of edges. Combinatorica 17(1), 1-9 (1997)

5. Alam, M.J., Brandenburg, F.J., Kobourov, S.G.: Straight-line grid drawings of 3-connected 1-planar graphs. In: Wismath, S., Wolff, A. (eds.) GD 2013. LNCS, vol. 8242, pp. 83-94. Springer, Heidelberg (2013)

6. Angelini, P., Di Battista, G., Didimo, W., Frati, F., Hong, S.H., Kaufmann, M., Liotta, G., Lubiw, A.: Large angle crossing drawings of planar graphs in subquadratic area. In: Márquez, A., Ramos, P., Urrutia, J. (eds.) EGC 2011. LNCS, vol. 7579, pp. 200-209. Springer, Heidelberg (2012)

7. Asano, K.: The crossing number of $K_{1,3, n}$ and $K_{2,3, n}$. J. of Graph Theory 10(1), 1-8 (1986)

8. Auer, C., Bachmaier, C., Brandenburg, F.J., Gleißner, A., Hanauer, K., Neuwirth, D., Reislhuber, J.: Recognizing outer 1-planar graphs in linear time. In: Wismath, S., Wolff, A. (eds.) GD 2013. LNCS, vol. 8242, pp. 107-118. Springer, Heidelberg (2013)

9. Auer, C., Brandenburg, F.J., Gleißner, A., Hanauer, K.: On sparse maximal 2-planar graphs. In: Didimo, W., Patrignani, M. (eds.) GD 2012. LNCS, vol. 7704, pp. 555-556. Springer, Heidelberg (2013)

10. Brandenburg, F.J., Eppstein, D., Gleißner, A., Goodrich, M.T., Hanauer, K., Reislhuber, J.: On the density of maximal 1-planar graphs. In: Didimo, W., Patrignani, M. (eds.) GD 2012. LNCS, vol. 7704, pp. 327-338. Springer, Heidelberg (2013)

11. Cheong, O., Har-Peled, S., Kim, H., Kim, H.S.: On the number of edges of fan-crossing free graphs. In: Cai, L., Cheng, S.-W., Lam, T.-W. (eds.) ISAAC 2013. LNCS, vol. 8283, pp. 163-173. Springer, Heidelberg (2013) 
12. Dehkordi, H.R., Eades, P.: Every outer-1-plane graph has a right angle crossing drawing. International J. on Computational Geometry and Appl. 22(6), 543-558 (2012)

13. Di Giacomo, E., Didimo, W., Eades, P., Liotta, G.: 2-layer right angle crossing drawings. Algorithmica 68(4), 954-997 (2014)

14. Di Giacomo, E., Didimo, W., Liotta, G., Meijer, H.: Area, curve complexity, and crossing resolution of non-planar graph drawings. Theory of Computing Syst. 49(3), 565-575 (2011)

15. Di Giacomo, E., Didimo, W., Liotta, G., Montecchiani, F.: h-quasi planar drawings of bounded treewidth graphs in linear area. In: Golumbic, M.C., Stern, M., Levy, A., Morgenstern, G. (eds.) WG 2012. LNCS, vol. 7551, pp. 91-102. Springer, Heidelberg (2012)

16. Di Giacomo, E., Didimo, W., Liotta, G., Montecchiani, F.: Area requirement of graph drawings with few crossings per edge. Computational Geometry 46(8), 909-916 (2013)

17. Dickerson, M., Eppstein, D., Goodrich, M.T., Meng, J.Y.: Confluent drawings: Visualizing non-planar diagrams in a planar way. J. of Graph Algorithms and Appl. 9(1), 31-52 (2005)

18. Didimo, W.: Density of straight-line 1-planar graph drawings. Information Processing Letters 113(7), 236-240 (2013)

19. Didimo, W., Eades, P., Liotta, G.: Drawing graphs with right angle crossings. Theor. Comput. Sci. 412(39), 5156-5166 (2011)

20. Didimo, W., Liotta, G.: The crossing angle resolution in graph drawing. In: Pach, J. (ed.) Thirty Essays on Geometric Graph Theory. Springer (2012)

21. Dujmović, V., Gudmundsson, J., Morin, P., Wolle, T.: Notes on large angle crossing graphs. Chicago J. on Theoretical Computer Science 2011 (2011)

22. Eades, P., Liotta, G.: Right angle crossing graphs and 1-planarity. Discrete Applied Mathematics 161(7-8), 961-969 (2013)

23. Eppstein, D., Goodrich, M.T., Meng, J.Y.: Confluent layered drawings. Algorithmica 47(4), 439-452 (2007)

24. Fox, J., Pach, J., Suk, A.: The number of edges in $k$-quasi-planar graphs. SIAM J. on Discrete Mathematics 27(1), 550-561 (2013)

25. Grigoriev, A., Bodlaender, H.L.: Algorithms for graphs embeddable with few crossings per edge. Algorithmica 49(1), 1-11 (2007)

26. Hong, S.H., Eades, P., Katoh, N., Liotta, G., Schweitzer, P., Suzuki, Y.: A linear-time algorithm for testing outer-1-planarity. In: Wismath, S., Wolff, A. (eds.) GD 2013. LNCS, vol. 8242, pp. 71-82. Springer, Heidelberg (2013)

27. Hong, S.-H., Eades, P., Liotta, G., Poon, S.-H.: Fáry's theorem for 1-planar graphs. In: Gudmundsson, J., Mestre, J., Viglas, T. (eds.) COCOON 2012. LNCS, vol. 7434, pp. 335-346. Springer, Heidelberg (2012)

28. Kaufmann, M., Ueckerdt, T.: The density of fan-planar graphs. CoRR abs/1403.6184 (2014), http://arxiv.org/abs/1403.6184

29. Korzhik, V.P., Mohar, B.: Minimal obstructions for 1-immersions and hardness of 1-planarity testing. J. of Graph Theory 72(1), 30-71 (2013)

30. Pach, J., Tóth, G.: Graphs drawn with few crossings per edge. Combinatorica 17(3), 427-439 (1997)

31. Schaefer, M.: The graph crossing number and its variants: A survey. Electronic J. of Combinatorics 20(2) (2013)

32. Suzuki, Y.: Re-embeddings of maximum 1-planar graphs. SIAM J. on Discrete Mathematics 24(4), 1527-1540 (2010)

33. Valtr, P.: On geometric graphs with no $k$ pairwise parallel edges. Discrete \& Computational Geometry 19(3), 461-469 (1998)

34. Garey, M., Johnson, D.: Crossing Number is NP-Complete. SIAM Journal on Algebraic Discrete Methods 4(3), 312-316 (1983), doi:10.1137/0604033 